\title{
A novel approach to basal-bolus insulin initiation in adults with newly diagnosed type 1 diabetes: an observational cohort study of a service redesign
}

\author{
HELEN E HOPKINSON, ${ }^{1}$ ANNA D WHITE, ${ }^{2}$ PETER NIGHTINGALE, ${ }^{3}$ PARTH NARENDRAN 4
}

\begin{abstract}
Aims: The National Institute for Health and Care Excellence (NICE) recommendation for insulin in newly diagnosed type 1 diabetes is a 'basal-bolus' regimen of prandial insulin with twice-daily basal insulin initiated at diagnosis. We developed an insulin initiation programme that embraces the contribution of endogenous insulin to glycaemic control in adults newly diagnosed with type 1 diabetes. Our aim was to embed carbohydrate counting skills and dose adjustment very early on to mitigate against the decline in glycaemic control that is commonly seen post honeymoon.

Methods: We designed a novel insulin initiation programme that focused initially on prandial insulin replacement using the lowest possible dose of basal insulin. The approach also facilitates carbohydrate counting and bolus insulin dose adjustment behaviours from diagnosis.

Results: Prior to implementing the new programme, the mean $\mathrm{HbA}_{1 \mathrm{c}}$ at 12 months was $64 \mathrm{mmol} / \mathrm{mol}(8.0 \%)(95 \% \mathrm{Cl}$ 60 to $69(7.6 \%$ to $8.4 \%))$. This reduced to $55 \mathrm{mmol} / \mathrm{mol}$ (7.1\%) $(95 \% \mathrm{Cl} 51$ to $58(6.9 \%$ to $7.4 \%)), p<0.001$ with the new programme. The improved $\mathrm{HbA}_{1 c}$ persisted to 3 years of follow-up $(p<0.001)$. There were no episodes of diabetic ketoacidosis or severe hypoglycaemia associated with this novel approach.

Conclusions: We suggest that using minimal basal insulin and focusing on prandial bolus insulin replacement in adults newly diagnosed with type 1 diabetes is safe, more physiological and may be better able to achieve lasting glycaemic control than the currently proposed national guidelines. This
\end{abstract}

Greater Glasgow and Clyde Health Board, Glasgow, UK

2 University of Glasgow, Glasgow, UK

3 University Hospitals Birmingham NHS Foundation Trust, Birmingham, UK

${ }^{4}$ University of Birmingham, UK

Address for correspondence: Dr Helen E Hopkinson Consultant Physician, Diabetes Department, Clinic L, New Victoria Hospital, Grange Road, Glasgow G42 9LF, UK

Email: helen.hopkinson@nhs.net

https://doi.org/10.15277/bjd.2018.175 approach will need to be tested formally in an adequately designed randomised controlled clinical trial.

Br J Diabetes 2018;18:71-75

Key words: Type 1 diabetes, outcome(s), basal-bolus, management, $\mathrm{HbA}_{1 \mathrm{c}}$, insulin, honeymoon, initiation, training, self-management.

\section{Introduction}

The recommended insulin regimen for adults in the UK with type 1 diabetes is twice daily basal insulin and multiple-dose prandial insulin before consuming carbohydrate-containing food. ${ }^{1}$ Current National Institute for Health and Care Excellence (NICE) guidelines (NG17) recommend this insulin regimen to be commenced at diagnosis. We propose that replacing insulin incrementally starting with an emphasis on prandial carbohydrate-adjusted dosing with minimal basal insulin initially may complement more effectively the deficits in beta cell function at the time of diagnosis with type 1 diabetes. Furthermore, we propose that focusing on carbohydrate counting skills at diagnosis promotes the embedding of self-management behaviours in adults with new type 1 diabetes.

This report describes the results of introducing a novel insulin initiation algorithm that focuses on replacement of prandial insulin over basal for adults newly diagnosed with type 1 diabetes in our clinic. We compared outcomes between two cohorts of our patients before and after the change in management.

\section{Methods}

Retrospective case notes review was performed from paper and electronic case records of all adult patients newly diagnosed with type 1 diabetes in a Scottish city hospital from January 2009 to December 2012 (Group A). After a change in the approach to insulin initiation, further review was performed from January 2013 to December 2015 (Group B).

Our pathway for assessment of adults with new type 1 diabetes already included avoidance of hospital admission if clinically appropriate and feasible. It was already established practice that general practitioners would refer directly to the diabetes centre rather than for acute admission, and the acute admissions unit was already diverting appropriate patients directly to the diabetes team. Patients 
were seen within 24 hours by a diabetes specialist nurse and by a diabetes specialist dietician either on the same visit or as soon as possible afterwards. Patients who had been admitted to hospital either due to established diabetic ketoacidosis (DKA) or presentation out of hours were seen on the ward or within 24 hours of hospital discharge. However, prior to January 2013, different insulin initiation regimens were employed at diagnosis of type 1 diabetes in adults, often depending on which member of the team saw the patient. Carbohydrate counting was not taught to anyone at diagnosis and this training was reserved until individuals could attend a structured education course (DAFNE at our centre) not before 12 months from diagnosis.

The approach to insulin initiation from January 2013 is summarised in the algorithm in Figure 1. Prandial insulin was either insulin lispro or insulin aspart, and basal was either human NPH or detemir. Education was individually delivered and tailored to the needs of the patient to achieve independent carbohydrate counting and insulin dose adjustment using ratios and correction doses. Blood glucose targets in line with NICE 2015 were emphasised at all visits and the use of correction doses using an insulin sensitivity factor was encouraged. The mantra was to focus on carbohydrate counting accuracy and to use the minimal amount of basal insulin to achieve good glycaemic control. Patients who commenced only prandial insulin with good glycaemic control were supplied with basal insulin and advised that, if their fasting blood glucose rose above a target of $7 \mathrm{mmol} / \mathrm{L}$, they should contact the clinic to initiate basal insulin. The long-term basal insulin regimen of choice was twice daily but, based on blood glucose requirements, this was initiated in small increments, initially once daily (usually at bedtime) with an explanation and anticipation of twice daily when required clinically. Insulin management was varied from the specified approach only if insulin was contraindicated due to pronounced 'honeymoon phase' or if the patient had literacy/numeracy issues that prevented carbohydrate counting. Individuals who required basal insulin at diagnosis but then developed partial remission (evidenced by reduced insulin requirements and hypoglycaemia) were managed by reduction and even withdrawal of basal insulin, but persistence with prandial insulin doseadjusted for carbohydrate consumption. A small number of patients without ketones and with only postprandial hyperglycaemia were managed initially on sulfonylurea therapy pending results of immune serology. In these patients the insulin algorithm was initiated as soon as clinically necessary.

Consultant review was arranged as soon as possible after diagnosis and always within 3 weeks of diagnosis, unchanged from the previous pathway. $\mathrm{HbA}_{1 \mathrm{c}}$ was measured 3-monthly at a consultantled clinic in the first 12 months. Education - where relevant covering driving, exercise, alcohol, drugs and pregnancy - was delivered 1:1 by diabetes educators at a pace suited to the patient's learning needs, in the same way as it was prior to the change in insulin initiation. A group education programme based on the Leicester Foundation Programme ${ }^{2}$ was offered over two half-days at around 3 months after diagnosis. Patients were advised to anticipate attending a DAFNE course at around 12 months from diagnosis or soon after that providing they had developed a requirement for basal insulin, ideally twice daily.

Baseline data for all adults diagnosed with type 1 diabetes from 2009 to 2012 (group A) included age, gender, insulin regimen, serial basal insulin dose, serial $\mathrm{HbA}_{1 \mathrm{c}}$, incidence of DKA and severe hypoglycaemia in the first 12 months. The same data were collected prospectively for the 2013-15 cohort (group B). Glutamic acid decarboxylase antibodies and/or islet antigen-2 antibodies were measured in the majority of more recent diagnoses, especially in patients with a less acute presentation or age $>25$ years. Patients without a classical presentation whose immune serology was negative were excluded from the analysis.

An attempt to investigate the proportion of people with partial remission was made based on $\mathrm{HbA}_{1 \mathrm{c}} \leq 58 \mathrm{mmol} / \mathrm{mol}(\leq 7.5 \%)$ at 12 months. We looked at a subgroup analysis of people in both groups using a basal bolus regimen (including prandial only) to compare mean $\mathrm{HbA}_{1 \mathrm{c}}$ and mean basal insulin doses in order to exclude bias from twice daily mixed insulin regimens.

Statistical analyses were performed using SPSS Statistics for Windows, Version 22.0 (IBM Corp, Armonk, New York, USA). As the $\mathrm{HbA}_{1 \mathrm{c}}$ values were positively skewed, they were log transformed prior to analysis and are reported as geometric means with $95 \%$ confidence intervals, medians and quartiles. $\mathrm{HbA}_{1 \mathrm{c}}$ values at different time points between the groups were compared using $t$ tests with 
equal variances not assumed. Basal insulin doses were compared using the Mann-Whitney test.

\section{Results}

From 2009 to 2012 (group A) there were 87 patients newly diagnosed with type 1 diabetes and we were able to collect 12-month data for 84 of them (2 DNA, 1 missing): 53.6\% men; mean (SEM) age 31.9 (13.8) years. From 2013 to 2015 (group B) there were 67 newly diagnosed adults with 12-month data available for 58 patients ( 6 moved away and 3 DNA): $51.7 \%$ men, mean (SEM) age 36.0 (13.7) years. Forty-one people have reached their two-year anniversary with data available for 36 (1 died, 2 DNA, 2 moved). Of the 17 people who have reached their 3-year anniversary, one has moved away and data are available for 16 .

The insulin regimen at 12 months is shown in Table 1. All individuals using a basal-bolus regimen in either group A or B were using exclusively analogue prandial insulin. Basal insulin subtypes were significantly different between the two groups, with the majority of people using NPH insulin in group B and fewer using analogue basal insulin.

There was no significant difference between the geometric mean $\mathrm{HbA}_{1 \mathrm{c}}$ of groups $\mathrm{A}$ and $\mathrm{B}$ at baseline, 3 months or 6 months: baseline group $A(n=66) 107 \mathrm{mmol} / \mathrm{mol}(11.9 \%)$, median and quartiles 113 (89-131); baseline group $B(n=60) 108 \mathrm{mmol} / \mathrm{mol}(12.0 \%)$, median and quartiles 108 (95-134); $\mathrm{p}=0.852$. Twelve-month $\mathrm{HbA}_{1 \mathrm{c}}$ was significantly improved in both groups from baseline, and was significantly lower in group B with mean 12-month $\mathrm{HbA}_{1 \mathrm{c}} 55$ $\mathrm{mmol} / \mathrm{mol}(7.1 \%)(95 \% \mathrm{Cl} 51$ to $58(6.9 \%$ to $7.4 \%))$ compared with $64 \mathrm{mmol} / \mathrm{mol}(8.0 \%)(95 \% \mathrm{Cl} 60$ to $69(7.6 \%$ to $8.4 \%))$ for group $\mathrm{A}(\mathrm{p}<0.001)$. Geometric mean $\mathrm{HbA}_{1 \mathrm{c}}$ tracked consistently within each cohort from year 1 onwards and in group $B$ remained below $58 \mathrm{mmol} / \mathrm{mol}(7.5 \%)$ out to 3 years $(n=16)$. The mean $\mathrm{HbA}_{1 \mathrm{c}}$ values at 2 and 3 years were significantly lower in group $B(p<0.001)$ (Figure 2).

Mean basal insulin dose at 12 months were significantly lower in group B. Some individuals were not requiring any basal insulin at 12 months (1 in group A and 4 in group B; not significantly different). The mean basal dose at 12 months was 18.2 units/day in group A compared with 14.2 units/day in group $B(p=0.034)$ and 0.27 units $/ \mathrm{kg} /$ day versus $0.18 \mathrm{units} / \mathrm{kg} /$ day, respectively $(p=0.007)$.

We looked at partial remission at 12 months: analysis of the individuals in each group with 12-month $\mathrm{HbA}_{1 \mathrm{c}}$ of $\leq 58 \mathrm{mmol} / \mathrm{mol}$ $(\leq 7.5 \%)$ revealed 37 people $(44.0 \%)$ in group $A$ and 40 people $(69.0 \%)$ in group $B(p=0.004)$, with mean $\mathrm{HbA}_{1 c}$ of $48 \mathrm{mmol} / \mathrm{mol}$ $(6.5 \%)$ in both subgroups (Figure 3). Comparison of the basal insulin doses for people in partial remission showed no significant difference between the groups.

Looking at those using a basal-bolus (including prandial only) regimen at 12 months, the mean 12-month $\mathrm{HbA}_{1 \mathrm{c}}$ was 63 $\mathrm{mmol} / \mathrm{mol}(7.9 \%)$ in group A and significantly lower in group B at $55 \mathrm{mmol} / \mathrm{mol}(7.2 \%)(p=0.008)$. The basal insulin dose at 12 months was not significantly different between groups $A$ and $B$ when comparing only those using basal-bolus regimens $(p=0.7)$. The mean 12-month $\mathrm{HbA}_{1 \mathrm{c}}$ for those in group $\mathrm{A}$ using biphasic insulin at 12 months was $73 \mathrm{mmol} / \mathrm{mol}(8.8 \%)$.
Table 1 Insulin regimen details at 12 months for two cohorts of adults with newly diagnosed type 1 diabetes. Group A were diagnosed between 2009 and 2012 and group B (2013-15) were managed on the new pathway

\begin{tabular}{|lll|}
\hline & $\begin{array}{l}\text { Group A } \\
(\mathbf{n = 8 4 )}\end{array}$ & $\begin{array}{l}\text { Group B } \\
(\mathbf{n}=58)\end{array}$ \\
\hline Biphasic insulin & $31(36.9 \%)$ & $3(5.2 \%)$ \\
\hline Basal bolus (glargine) & $31(36.9 \%)$ & $5(8.6 \%)$ \\
\hline Basal bolus (detemir) & $6(7.1 \%)$ & $9(15.5 \%)$ \\
\hline Basal bolus NPH & $14(16.7 \%)$ & $35(60.3 \%)$ \\
\hline Prandial only & $1(1.2 \%)$ & $4(6.9 \%)$ \\
\hline Oral agents (GAD positive) & $1(1.2 \%)$ & $2(3.4 \%)$ \\
\hline
\end{tabular}

Figure 2. Tracking of geometric mean $\mathrm{HbA}_{1 c}$ over 60 months in two cohorts of adults newly diagnosed with type 1 diabetes with $95 \% \mathrm{Cl}$. There is a greater improvement in the carbohydrate counting group B. The benefit is sustained over 3 years in comparison with group A. There is no statistical difference between the groups at baseline, 3 and 6 months. The values at 12,24 and 36 months are significantly different between groups $A$ and $B$ (all $\mathrm{p}<0.001)$

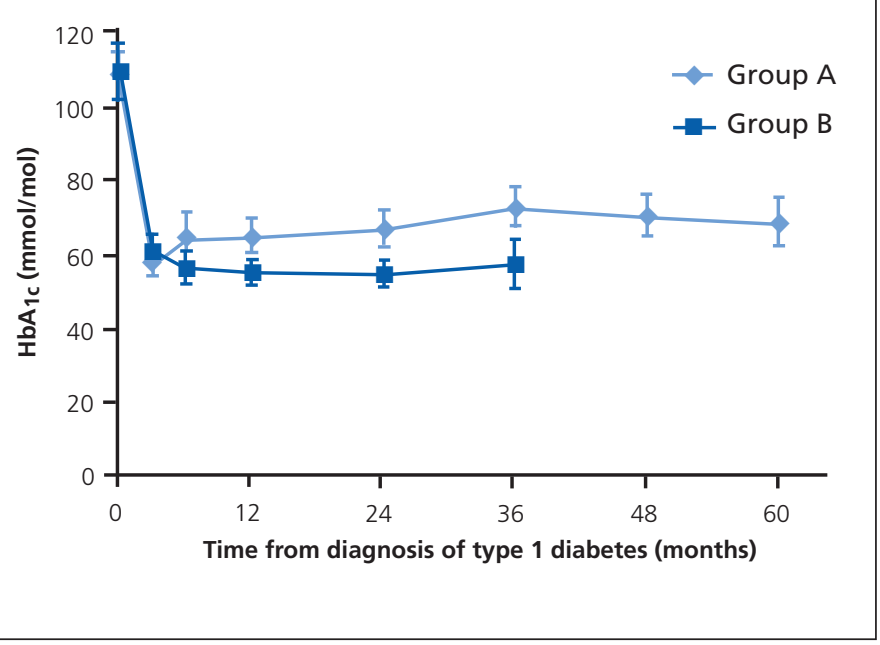

Excluding DKA at presentation, in the first year of diagnosis six episodes of DKA were recorded for group A (2 patients) and no episodes were recorded for group $B$. There were no reports of severe hypoglycaemia in either group; the incidence of mild hypoglycaemia was not accurately recorded.

The number of patients who participated in structured education was greater in group B. In group A, eight people (9.5\%) participated in the Foundation Programme (FP) and 14 people (16.7\%) completed a DAFNE course, with an average time from diagnosis to course of 16 months for FP and 47 months for DAFNE. In group B, 
Figure 3. Proportion of newly diagnosed adults with type 1 diabetes achieving target $\mathrm{HbA}_{1 \mathrm{c}} 12$ months after diagnosis, shown in two groups representing different insulin initiation regimens. Group A includes a mixture of people on twice-daily mixed insulin and basal bolus regimens without carbohydrate counting. For people in group B who were carbohydrate counting from diagnosis and used minimal basal insulin, the $\mathrm{HbA}_{1 \mathrm{c}}$ at 12 months was more likely to be at target. The difference between the proportions in each group achieving $\leq 48 \mathrm{mmol} / \mathrm{mol}(\leq 6.5 \%)$ was not significant $(p=0.122)$, but significantly more people achieved $\leq 58 \mathrm{mmol} / \mathrm{mol}(\leq 7.5 \%)$ in group B $(p=0.004)$.

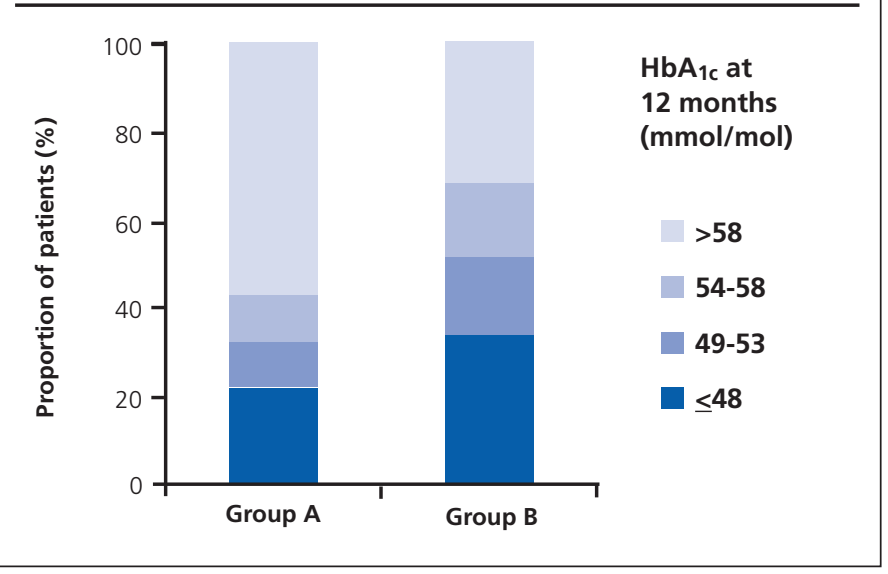

15 people (25.9\%) participated in the FP and seven (12.1\%) in a DAFNE course, with average time from diagnosis to course of 7 and 19 months, respectively.

\section{Discussion}

This analysis of a service redesign suggests that our novel approach to basal-bolus insulin initiation for adults with newly diagnosed type 1 diabetes contributes to significantly improved glycaemic outcomes that are sustained for 3 years. We propose that focusing on replacement of prandial insulin and using minimal basal insulin when exogenous insulin production is still present makes physiological sense. There are three reasons for this proposal. First, the first-phase insulin response is the most prominent secretory defect at diagnosis, and any residual beta cell function is capable of supporting the less demanding basal insulin requirement. ${ }^{3,4}$ This is an important principle behind mealstimulated tests of beta cell function that are employed to study the natural history of type 1 diabetes, where a meal-stimulated measure of beta cell function is used in preference to a basal insulin estimate. ${ }^{5}$ Therefore, it is more physiologically appropriate to concentrate on replacing prandial insulin in favour of - or in combination with - basal insulin. Second, such an approach will embed accurate carbohydrate counting and bolus dose adjustment behaviours. Inappropriately adjusted basal insulin can mask inaccuracies in carbohydrate counting and bolus dose adjustments, thus interfering with patient training at this critical period.
Third, inappropriately excessive exogenous insulin replacement on top of a recovering capacity for endogenous insulin production can lead to hypoglycaemia and early negative experiences for patients - powerful behavioural distraction from the goal of tight blood glucose targets. ${ }^{6}$

We propose the strengths of this model are that it provides a practical platform on which to educate patients around carbohydrate counting. In the early weeks and months of adult new type 1 diabetes it can be difficult to embed insulin self-management behaviours due to the confounding effects of endogenous insulin production. After the initial crisis of hyperglycaemia in newly diagnosed type 1 diabetes has been successfully managed, in most people there is some residual insulin secretory capacity. ${ }^{7}$ We have observed in clinical practice that the beta cell deficit is most prominently unmasked following a carbohydrate challenge rather than in the fasting state. Presumably this is because persisting beta cell function can cover insulin requirements in the fasting state. Our model minimises the early use of exogenous basal insulin so that endogenous insulin reserve is utilised to cover basal requirement, either in its entirety or, more commonly, in part together with exogenous supplementation.

Supporting prandial insulin requirements provides real-time feedback to the patient on the accuracy of carbohydrate counting. The presence of exogenously administered basal insulin in line with current guidelines (using weight-based doses or algorithms such as 1 unit per hour) may compensate for inaccuracy of carbohydrate counting. This can reduce the need for prandial dose adjustment accuracy and obfuscate the learning that all carbohydrate requires insulin cover or, worse still, provides a subtle but constant drive for hypoglycaemia that discourages the use of bolus insulin.

The approach of prandial replacement is also ideally suited to managing the changing insulin requirement around the honeymoon phase. This can often be a confusing time for people with diabetes. The newly diagnosed adult may develop the idea that diabetes is easy to manage and fail to engage fully with the behaviours and concepts needed. Once the honeymoon phase ends, there is an urgent need to make significant changes and embed new behaviours in the context of high blood glucose readings which in themselves make the individual feel unwell. Overall, diabetes control can deteriorate significantly at this time. Our data demonstrate that this novel insulin initiation regimen mitigates against the post-honeymoon phenomenon. A rise in $\mathrm{HbA}_{1 c}$ over the first 12 months was seen in group $A$ but not in group $B$.

Maintaining good glycaemic control at 12 months is vital as reports suggest $\mathrm{HbA}_{1 \mathrm{c}}$ at 12 months after diagnosis predicts the level of glycaemic control that the person is likely to achieve in future years.8,9

Whilst there could be a perceived increased risk of DKA in people who are using minimal amounts of basal insulin, our study showed no such episodes in the 109 person-years of follow-up. One explanation for this observation could be that endogenous insulin production has been better preserved because protecting residual beta cells from the demands of prandial insulin secretion may support survival. ${ }^{10}$ Recovery of beta cell function contributes to the honeymoon period, ${ }^{11}$ and our $\mathrm{HbA}_{1 \mathrm{c}}$ data suggest a higher proportion of people still in the honeymoon phase at 1 year in group $B$, consistent with the suggestion that accurate prandial insulin replacement 
can modulate the natural history of beta cell loss.

We did not report total daily insulin doses due to the tendency for clinicians to note ratios rather than doses and we acknowledge this as a weakness in our report. Reduced use of biphasic insulin regimens in group B may have contributed to the improved outcomes for this group. However, looking only at those on basal-bolus regimens, the 12-month $\mathrm{HbA}_{1 c}$ was significantly lower in group $B$ than group $A$ with no significant difference in the mean basal insulin dose at 12 months. We propose this demonstrates an additional benefit of our novel approach beyond that associated with the change to an MDI regimen.

The study is weakened by the design being a retrospective analysis, and the fact that background improvement in diabetes care provision may have contributed to the improvement over time. Our diabetes educators anecdotally report that people with new type 1 diabetes appear to become competent, confident and independent after fewer visits on the new pathway than previously, and that this appears to be related to fewer problems with hypoglycaemia. Unfortunately, as this was a retrospective review of notes we were unable to compare accurately either the number of visits or the incidence of non-severe hypoglycaemia. The majority of people in group B were using NPH basal insulin, therefore the putative benefit of analogue basal insulin is not a confounding factor in our data.

There was some loss to follow-up and data that were not available for a full analysis. However, the uptake of group structured education within the recommended 1 year after diagnosis was disappointingly low in both groups and therefore this is not a confounding factor in the sustained improvement in glycaemic control seen in group B. Perhaps delivering detailed education early after diagnosis provides a more solid grounding in dose adjustment skills, thereby reducing the development of maladaptive behaviours that can be difficult to change later on. People may be more prepared to take time out from their busy lives to learn skills at diagnosis than they appear to be later. The difficulties in persuading people to attend structured education years after diagnosis are well documented, and guidelines advise delaying formal structured education until post-honeymoon. However, our findings suggest that it is feasible and effective to teach carbohydrate-counting and dose-adjustment skills from diagnosis by using the minimal amount of basal insulin and making use of endogenous reserve. In our model, group structured education such as DAFNE is relevant and beneficial in the first 1-3 years after diagnosis for consolidating and refreshing self-management skills when endogenous insulin production has ceased.

To our knowledge, there are no other studies that have reported on a prandial insulin replacement regimen at onset of type 1 diabetes in adults. A recent survey of UK healthcare professionals involved in type 1 diabetes care illustrated the lack of consistency in clinical practice. ${ }^{12}$ We propose this observation stems from the lack of efficacy of the various options currently in use, together with a lack of evidence that any particular insulin regimen is associated with better early and delayed clinical outcomes. Whilst the results of our study are interesting, it remains a retrospective analysis and cannot be used to recommend a wider change in routine practice. We propose a randomised controlled trial to validate these findings in a prospective manner.

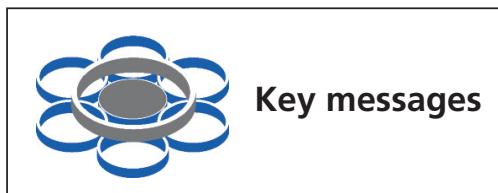

- Establishing effective self management behaviours from diagnosis of Type 1 diabetes in adults can be difficult due to confounding effects of persisting endogenous insulin.

- Endogenous insulin can be used to contribute to basal insulin requirement.

- Initially using prandial insulin and carbohydrate counting education together with the minimum possible basal insulin in new Type 1 diabetes in adults makes physiological sense.

- In this observational cohort study, glycaemic control is better at 12 months and sustained for 3 years with no episodes of DKA

Acknowledgements: With thanks to diabetes educators Amy Cowan, Caroline Gillies, Margaret Kyle, Carol McMaster

\section{Conflict of interest: None}

\section{Funding: None}

\section{References}

1. National Institute for Health and Care Excellence (NICE) Guideline NG 17 Type 1 diabetes in adults: diagnosis and management. Updated July 2016. https://www.nice.org.uk/guidance/ng17 (accessed 5 July 2017).

2. Leicester Foundation Group. Successful implementation of structured education for newly diagnosed type 1 diabetes. Practical Diabetes 2012;29:149-52. https://doi.org/10.1002/pdi.1679

3. Eisenbarth GS. Type 1 diabetes mellitus. N Engl J Med 1986;314:13608. https://doi.org/10.1056/NEJM198605223142106

4. Sosenko JM, Skyler JS, Beam CA et al. Type 1 Diabetes TrialNet and Diabetes Prevention Trial-Type 1 Study Groups. Acceleration of the loss of the first-phase insulin response during the progression to type 1 diabetes in diabetes prevention trial-type 1 participants. Diabetes 2013; 62:417983. https://doi.org/10.2337/db13-0656

5. Greenbaum CJ, Harrison LC. Immunology of Diabetes Society. Guidelines for intervention trials in subjects with newly diagnosed type 1 diabetes. Diabetes 2003;52:1059-65. https://doi.org/10.2337/diabetes.52.5.1059

6. Bloomgarden Z. Fear of hypoglycemia. J Diabetes 2017;9:108-10. https://doi.org/10.1111/1753-0407.12491

7. Greenbaum CJ, Beam CA, Boulware D et al. Fall in C-peptide during first 2 years from diagnosis: evidence of at least two distinct phases from composite type 1 Diabetes TrialNet data. Diabetes 2012;61:2066-73. https://doi.org/10.2337/db11-1538

8. Jackson C, Wernham EM, Elder CJ, Wright NP. Early glycaemic control is predictive of longterm control: a retrospective observational study. Practical Diabetes 2013;30:16-18. https://doi.org/10.1002/pdi.1734

9. Jorde R, Sundesfjord J. Intra-individual variability and longitudinal changes in glycaemic control in patients with type 1 diabetes mellitus. Diabet Med 2000;17:451-6. https://doi.org/10.1046/j.1464-5491.2000.00295.x

10. Chmelova H, Cohrs CM, Chouinard JA, et al. Distinct roles of beta cell mass and function during type 1 diabetes onset and remission. Diabetes 2015;64:2148-60. https://doi.org/10.2337/db14-1055

11. Hanan A, Gottlieb P. The honeymoon phase: intersection of metabolism and immunology. Curr Opin Endocrinol Diabetes Obes 2009;16:286-92. https://doi.org/10.1097/MED.0b013e32832e0693

12. Taylor $\mathrm{C}$, Hopkinson $\mathrm{H}$, Elliott J. Which insulin regimens are used in adults newly diagnosed with type 1 diabetes? Results of an electronic survey of healthcare professionals in the UK and Ireland. $\mathrm{Br} J$ Diabetes 2016;16:A1-4. https://doi.org/10.15277/bjd.2016.085 